Research Article

\title{
Robust Output Synchronization of Arrays of Chaotic Sprott Circuits
}

\author{
Ernesto V. Gonzalez Solis and David I. Rosas Almeida \\ Facultad de Ingeniería, Universidad Autónoma de Baja California, Blvd. Benito Juárez s/n, Mexicali, BC, Mexico \\ Correspondence should be addressed to David I. Rosas Almeida; drosas@uabc.edu.mx
}

Received 31 January 2017; Accepted 25 July 2017; Published 23 August 2017

Academic Editor: Giampaolo Cristadoro

Copyright (c) 2017 Ernesto V. Gonzalez Solis and David I. Rosas Almeida. This is an open access article distributed under the Creative Commons Attribution License, which permits unrestricted use, distribution, and reproduction in any medium, provided the original work is properly cited.

\begin{abstract}
This article presents a technique for synchronizing arrays of a class of chaotic systems known as Sprott circuits. This technique can be applied to different topologies and is robust to parametric uncertainties caused by tolerances in the electronic components. The design of coupling signals is based on the definition of a set of functionals which depend on the errors between the outputs of the nodes and the errors between the output of a reference system and the outputs of the nodes. When there are no parametric uncertainties, we establish a criterion to design the coupling signals using only one state variable of each system. When the parametric uncertainties are present, we add a robust observer and a low pass filter to estimate the perturbation terms, which are subsequently compensated through the coupling signals, resulting in a robust closed loop system. The performance of the synchronization technique is illustrated by real-time simulations.
\end{abstract}

\section{Introduction}

Synchronization is a dynamical behavior that two or more systems exhibit when a correlated motion between them is established [1]. This phenomenon appears very often in nature, but it can also be forcibly induced by introducing some coupling elements or input signals in a convenient way. This last case is often denoted as controlled synchronization because it becomes a control objective [2].

Controlled synchronization can be achieved in dynamical systems on chaotic regime [3-8]. However, in practice, chaos synchronization is far from being straightforward because different aspects, as the presence of external disturbances and parameter uncertainties, affect significantly the ability of systems to synchronize. Therefore, robustness is a very desirable property of a synchronization approach.

Chaos synchronization has important applications, for example, in private communication systems; see, for example, $[4,5,9]$, where simplicity is always a desirable characteristic to consider in a practical implementation. As shown by Sprott [10], a practical chaos generator can be constructed with a simple electronic circuit with nonlinear terms as piecewise linear functions.
Recently, some synchronization techniques for Sprott circuits and other kinds of systems have been proposed for the master/slave scheme and for arrays or networks of them. Some important papers are [5, 9, 11-14].

A proposal to synchronize Sprott circuits and their application in secure communication are presented in [4]. Here a proportional-integral-derivative (PID) control scheme is used to solve the synchronization problem of identical chaotic systems and without disturbances under the master/slave configuration. To verify the system performance, basic electronic components are used to implement the proposed chaotic secure communication system. Something similar is presented in [5], but here, the information is incorporated as a disturbance, which is recovered through the equivalent control produced by a variable structure control.

A robust algorithm to synchronize Sprott circuits, under the master/slave configuration, using sliding mode control is presented in [14]. The objective is to obtain identical synchronization between the master and slave systems in spite of existence of external disturbances. An important condition is that both systems must have the same nonlinear function. The coupling signal contains a term to linearize the error dynamics and add a discontinuous part that gives 
robustness to the closed loop system. With the same objective and conditions, a technique to synchronize two Sprott circuits in finite time is presented in [15]. An important characteristic in this work is that the slave system incorporates two control inputs. These are designed based on variable structure control and depend on all of states of the systems. As in [14], the master and the slave systems must have the same nonlinear function.

In [16], a technique to synchronize arrays of dynamical systems is presented. The arrays are formed by uncertain nonlinear second-order systems where only the generalized position is available. The synchronization technique can be applied to many array topologies where the connections can be unidirectional or bidirectional with different weights; this produces a connection matrix that it is not necessarily symmetric. The design of the coupling signals is based on a robust discontinuous controller and on an exact deriver that estimates the velocity of each node. This is an interesting proposal but it is not applicable to Sprott circuits.

In this paper, we present a technique, based on [16], for synchronizing arrays of a class of chaotic systems known as Sprott circuits. This technique can be applied to different topologies and is robust to parametric uncertainties caused by tolerances in the electronic components. The design of coupling signals is based on the definition of a set of functionals, which depend on the errors between the outputs of the nodes and on the errors between the output of a reference system and the outputs of the nodes; when there are no parametric uncertainties, we establish a criterion to design the coupling signals using only one state variable of each system. When the parametric uncertainties are present, we add a robust observer and a low pass filter to estimate the perturbation terms, which are subsequently compensated through the coupling signals, resulting in a robust closed loop system. The performance of the synchronization technique is illustrated by experimental results.

The organization of the paper is as follows. Section 2 includes some preliminary definitions and the statement of the synchronization objective. In Section 3, the synchronization technique is described. Here, a sufficient condition on synchronizability is established. Also, in this section, a methodology to design the coupling signals is presented. In Section 4, the performance of the synchronization technique is shown through a real-time simulation where an array formed by four nodes and a reference system is synchronized. Finally, in Section 5, some conclusions are presented.

\section{Preliminary Definitions and Problem Statement}

Consider $k$ nonlinear dynamical systems, known as Sprott circuits [10], modeled by

$$
\Sigma_{i}: \frac{d^{3} y_{i}}{d t^{3}}+\left(a_{0}+\Delta_{a_{i}}\right) \frac{d^{2} y_{i}}{d t^{2}}+\frac{d y_{i}}{d t}=G_{l_{i}}\left(y_{i}\right)+u_{i},
$$

for $i=1, \ldots, k$, where $G_{l_{i}}\left(y_{i}\right)$ is one of the following functions:

$$
G_{a_{i}}\left(y_{i}\right)=\left(b_{0}+\Delta_{b_{i}}\right)\left|y_{i}\right|-\left(c_{0}-\Delta_{c_{i}}\right)
$$

$$
\begin{aligned}
& G_{b_{i}}\left(y_{i}\right)=-\left(b_{0}+\Delta_{b_{i}}\right) \max \left(y_{i}, 0\right)+\left(c_{0}-\Delta_{c_{i}}\right), \\
& G_{c_{i}}\left(y_{i}\right)=\left(b_{0}+\Delta_{b_{i}}\right) y_{i}-\left(c_{0}-\Delta_{c_{i}}\right) \operatorname{sign}\left(y_{i}\right), \\
& G_{d_{i}}\left(y_{i}\right)=-\left(b_{0}+\Delta_{b_{i}}\right) y_{i}+\left(c_{0}-\Delta_{c_{i}}\right) \operatorname{sign}\left(y_{i}\right),
\end{aligned}
$$

where $y_{i}$ is the output system; $a_{0}, b_{0}$, and $c_{0}$ are nominal values proposed in [10], for which system (1) presents chaotic behavior; $\Delta_{a_{i}}, \Delta_{b_{b}}$, and $\Delta_{c_{i}}$ are parametric variations due to tolerances in electronic components; and $u_{i}$ are the coupling signals to be designed for each node.

A state space representation of system (1), taking the state variables as $x_{1_{i}}=y_{i}, x_{2_{i}}=d y_{i} / d t$, and $x_{3_{i}}=d^{2} y_{i} / d t^{2}$, is

$$
\begin{aligned}
\Sigma_{i}: & {\left[\begin{array}{c}
\dot{x}_{1_{i}} \\
\dot{x}_{2_{i}} \\
\dot{x}_{3_{i}}
\end{array}\right] } \\
& =\left[\begin{array}{c}
x_{2_{i}} \\
x_{3_{i}} \\
-a_{0} x_{3_{i}}-x_{2_{i}}+G_{l_{i}}\left(x_{1_{i}}\right)+\gamma_{i}\left(x_{1_{i}}\right)+u_{i}
\end{array}\right], \\
y_{i} & =x_{1_{i}},
\end{aligned}
$$

where $\gamma_{i}\left(x_{1_{i}}\right)$ is a disturbance term due to parametric variations; it is unknown but bounded. Also, consider a reference system given by

$$
\begin{aligned}
\Sigma_{r}:\left[\begin{array}{c}
\dot{x}_{1_{r}} \\
\dot{x}_{2_{r}} \\
\dot{x}_{3_{r}}
\end{array}\right] & =\left[\begin{array}{c}
x_{2_{r}} \\
x_{3_{r}} \\
-a_{0} x_{3_{r}}-x_{2 r}+G_{l_{r}}\left(x_{1_{r}}\right)+\gamma_{r}\left(x_{1_{r}}\right)
\end{array}\right] . \\
y_{r} & =x_{1_{r}} .
\end{aligned}
$$

System (4) is defined in the same way of system (3), but in this case a coupling signal is not present.

Let us consider $k$ functionals $\epsilon_{i}: Y_{1} \times Y_{2} \times \cdots \times Y_{k} \times Y_{r} \rightarrow$ $\mathfrak{R}, i=1, \ldots, k$. Here $Y_{i}$ are the sets of all output functions.

Definition 1. One says that outputs $y_{1}(t), \ldots, y_{k}(t), y_{r}(t)$ of the systems $\Sigma_{1}, \ldots, \Sigma_{k}, \Sigma_{r}$ are synchronized with respect to the functionals $\epsilon_{1}, \ldots, \epsilon_{k}$ if

$$
\epsilon_{i}\left(y_{i}(t), \ldots, y_{k}(t), y_{r}(t)\right)=0, \quad i=1, \ldots, k .
$$

Definition 2. One says that outputs $y_{1}(t), \ldots, y_{k}(t), y_{r}(t)$ of the systems $\Sigma_{1}, \ldots, \Sigma_{k}, \Sigma_{r}$ with initial conditions $\left(x_{1_{1}}(0), x_{2_{1}}(0), x_{3_{1}}(0)\right)^{T}, \ldots,\left(x_{1_{k}}(0), x_{2_{k}}(0), x_{3_{k}}(0)\right), \quad\left(x_{1_{1}}(0)\right.$, $\left.x_{2_{1}}(0), x_{3_{1}}(0)\right)^{T}$ are asymptotically synchronized with respect to the functionals $\epsilon_{1}, \ldots, \epsilon_{k}$ if

$$
\lim _{t \rightarrow \infty} \epsilon_{i}\left(y_{i}(t), \ldots, y_{k}(t), y_{r}(t)\right)=0, \quad i=1, \ldots, k .
$$

Definition 3. One says that outputs $y_{1}(t), \ldots, y_{k}(t), y_{r}(t)$ of the systems $\Sigma_{1}, \ldots, \Sigma_{k}, \Sigma_{r}$ with initial conditions $\left(x_{1_{1}}(0), x_{2_{1}}(0), x_{3_{1}}(0)\right)^{T}, \ldots,\left(x_{1_{k}}(0), x_{2_{k}}(0), x_{3_{k}}(0)\right)^{T},\left(x_{1_{1}}(0)\right.$, $\left.x_{2_{1}}(0), x_{3_{1}}(0)\right)^{T}$ are approximately synchronized with respect 
to the functionals $\epsilon_{1}, \ldots, \epsilon_{k}$ if there is an $\varepsilon>0$ such that

$$
\left|\epsilon_{i}\left(y_{i}(t), \ldots, y_{k}(t), y_{r}(t)\right)\right| \leq \varepsilon, \quad i=1, \ldots, k .
$$

Finally, we define the concept of connection graph. A connection graph is a figure that describes the presence or absence of couplings among reference system (4) and nodes (3) in an array of systems. Figure 1 shows an example of a connection graph with three nodes $\Sigma_{i}$, orange spheres, and a reference system $\Sigma_{r}$, green sphere. Here solid lines denote connections among nodes and dashed lines connect the reference system with nodes. Both kinds of couplings have a particular direction represented by an arrow. An arrow from $\Sigma_{i}$ to $\Sigma_{j}$ indicates that the $j$ th node has access to the output $y_{i}$ of $i$ th node. It is important to notice that couplings between nodes may be bidirectional; however, couplings from the reference system to nodes are unidirectional.

2.1. The Synchronization Objective. Based on the precedent definitions, the problem is to design the coupling signals $u_{i}$ such that the objectives

$$
\begin{aligned}
& \lim _{t \rightarrow \infty}\left\|y_{i}(t)-y_{j}(t)\right\|=0, \quad \forall i, j \in\{1, \ldots, k\}, \quad i \neq j, \\
& \lim _{t \rightarrow \infty}\left\|y_{r}(t)-y_{i}(t)\right\|=0, \quad \forall i \in\{1, \ldots, k\}
\end{aligned}
$$

are satisfied.

Define the error variables between the reference system and the nodes as

$$
e_{i, r}=y_{i}-y_{r}
$$

Also, define errors between nodes as

$$
e_{i, j}=y_{i}-y_{j}, \quad i \neq j,
$$

for $i, j=1, \ldots, k$.
Equations (9) and (10) define $k(k+1) / 2$ independent errors which we want to regulate around zero with only $k$ coupling signals. For that, a set of functionals $\epsilon_{1}, \ldots, \epsilon_{k}$ is defined as a linear combination of errors $e_{i, r}$ and $e_{i, j}$ defined by

$$
\begin{gathered}
\epsilon_{1}=\alpha_{1, r} e_{1, r}+\beta_{1,2} e_{1,2}+\cdots+\beta_{1, j} e_{1, j}+\cdots+\beta_{1, k} e_{1, k}, \\
\epsilon_{2}=\alpha_{2, r} e_{2, r}+\beta_{2,1} e_{2,1}+\cdots+\beta_{2, j} e_{2, j}+\cdots+\beta_{2, k} e_{2, k}, \\
\vdots \\
\epsilon_{i}=\alpha_{i, r} e_{i, r}+\beta_{i, 1} e_{i, 1}+\cdots+\beta_{i, j} e_{i, j}+\cdots+\beta_{i, k} e_{i, k}, \\
\vdots \\
\epsilon_{k}=\alpha_{k, r} e_{k, r}+\beta_{k, 1} e_{k, 1}+\cdots+\beta_{k, j} e_{k, j}+\cdots \\
+\beta_{k, k-1} e_{k, k-1},
\end{gathered}
$$

where $\alpha_{i, r}$ and $\beta_{i, j}$ are constants. Analyzing the $i$ th functional, we can see that the constant $\alpha_{i, r}$ always corresponds to error $e_{i, r}$ whose composition requires that reference system output $y_{r}$ be available, which is possible according to the connection graph definition whenever a dashed line arrow exists between $i$-node and the reference system. If a coupling actually exists, the elements $\alpha_{i, r}$ take the value of one; or else these elements are zero. Similarly, constant $\beta_{i, j}$ indicates presence or absence of solid lines from the $j$-node to the $i$-node.

A matrix form of (11) is

$$
\epsilon=\mathscr{B} y-\mathscr{A} y_{r}
$$

where 


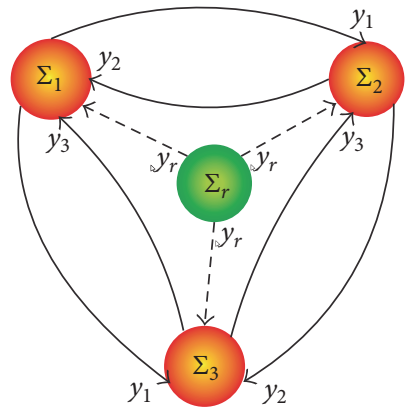

FIgURE 1: A connection graph. Example of full chain interconnection scheme.

$y_{r} \in \mathfrak{R}$, and all vectors and matrices have suitable dimensions.

Theorem 4. If vector $\mathscr{A} \neq 0$, matrix $\mathscr{B}$ is not singular, and the functionals (11) are zero, then the nodes (3) and the reference system (4) are synchronized according to Definition 1.

Proof. The proof of this theorem is in [17].

Note that if $\epsilon$ is not zero, then the synchronization objective given by Definition 2 can be attained if the coupling signals $u_{i}$ are designed such that these signals make $\epsilon$ converge to zero. This design is detailed in the next section. In practice, due to nonidealities in actual systems, we expect an approximate synchronization as established in Definition 3.

\section{Design of the Coupling Signals}

To design the coupling signals $u_{i}$, we define the state variables

$$
\begin{aligned}
& \epsilon=\mathscr{B} x_{1}-\mathscr{A} x_{1_{r}}, \\
& z=\mathscr{B} x_{2}-\mathscr{A} x_{2_{r}}, \\
& w=\mathscr{B} x_{3}-\mathscr{A} x_{3_{r}},
\end{aligned}
$$

whose dynamics are given by

$$
\begin{aligned}
\dot{\epsilon}= & z, \\
\dot{z}= & w, \\
\dot{w}= & -A_{0} w-z+\mathscr{B} G_{l}\left(x_{1}\right)-\mathscr{A} G_{l_{r}}\left(x_{1_{r}}\right)+\mathscr{B} \Gamma\left(x_{1}\right) \\
& -\mathscr{A} \gamma_{r}\left(x_{1_{r}}\right)+\mathscr{B} U,
\end{aligned}
$$

where

$$
\begin{aligned}
A_{0} & =\operatorname{diag}\left\{a_{0}\right\} \\
G_{l}\left(x_{1}\right) & =\left[\begin{array}{lllll}
G_{l_{1}}\left(x_{1_{1}}\right) & G_{l_{2}}\left(x_{1_{2}}\right) & \cdots & G_{l_{k}}\left(x_{1_{k}}\right)
\end{array}\right]^{T}, \\
\Gamma\left(x_{1}\right) & =\left[\begin{array}{llll}
\gamma_{1}\left(x_{1_{1}}\right) & \gamma_{2}\left(x_{1_{2}}\right) & \cdots & \gamma_{k}\left(x_{1_{k}}\right)
\end{array}\right]^{T}, \\
U & =\left[\begin{array}{llll}
u_{1} & u_{2} & \cdots & u_{k}
\end{array}\right]^{T}, \\
x_{1} & =\left[\begin{array}{llll}
x_{1_{1}} & x_{1_{2}} & \cdots & x_{1_{k}}
\end{array}\right]^{T},
\end{aligned}
$$

$$
\begin{aligned}
& x_{2}=\left[\begin{array}{llll}
x_{2_{1}} & x_{2_{2}} & \cdots & x_{2_{k}}
\end{array}\right]^{T}, \\
& x_{3}=\left[\begin{array}{llll}
x_{3_{1}} & x_{3_{2}} & \cdots & x_{3_{k}}
\end{array}\right]^{T} .
\end{aligned}
$$

Now the problem is to design a control vector $U$ to stabilize the origin of system (15). To do this, it is important to consider the following:

(1) System (15) has a linear part and nonlinear part that only depends on $x_{1}$; the term $\mathscr{B} G_{l}\left(x_{1}\right)-\mathscr{A} G_{l_{r}}\left(x_{1_{r}}\right)$ is well known.

(2) Matrix $\mathscr{B}$ is invertible.

(3) The Sprott systems are electronic circuits; therefore all state variables are available; then we can use a state observer to estimate the term $\mathscr{B} \Gamma\left(x_{1}\right)-\mathscr{A} \gamma_{r}\left(x_{1_{r}}\right)$, and we compensate it through $U$.

We propose the control input $U$ given by

$$
\begin{aligned}
U & =\mathscr{B}^{-1}\left(-K \epsilon-\mathscr{B} G_{l}\left(x_{1}\right)+\mathscr{A} G_{l_{r}}\left(x_{1_{r}}\right)-\mathscr{B} \widetilde{\Gamma}\left(x_{1}\right)\right. \\
& \left.+\mathscr{A} \widetilde{\gamma}_{r}\left(x_{1_{r}}\right)\right)
\end{aligned}
$$

where $\widetilde{\Gamma}\left(x_{1}\right)$ and $\widetilde{\gamma}_{r}\left(x_{1_{r}}\right)$ are the asymptotic estimates of $\Gamma\left(x_{1}\right)$ and $\gamma\left(x_{1_{r}}\right)$, respectively. $K$ is a constant diagonal matrix that will be designed.

Substituting (17) in (15), we have

$$
\begin{aligned}
\dot{\epsilon}= & z \\
\dot{z}= & w \\
\dot{w}= & -K \epsilon-z-A_{0} w+\mathscr{B}\left(\Gamma\left(x_{1}\right)-\widetilde{\Gamma}\left(x_{1}\right)\right) \\
& +\mathscr{A}\left(\tilde{\gamma}_{r}\left(x_{1_{r}}\right)-\gamma_{r}\left(x_{1_{r}}\right)\right) .
\end{aligned}
$$

System (18) is a disturbed linear system with a vanish disturbance; the disturbance is

$$
\mathscr{B}\left(\Gamma\left(x_{1}\right)-\widetilde{\Gamma}\left(x_{1}\right)\right)+\mathscr{A}\left(\tilde{\gamma}_{r}\left(x_{1_{r}}\right)-\gamma_{r}\left(x_{1_{r}}\right)\right),
$$

and the linear part has the form

$$
\left[\begin{array}{c}
\dot{\epsilon}_{1} \\
\vdots \\
\dot{\epsilon}_{k} \\
\dot{z}_{1} \\
\vdots \\
\dot{z}_{k} \\
\dot{w}_{1} \\
\vdots \\
\dot{w}_{k}
\end{array}\right]
$$




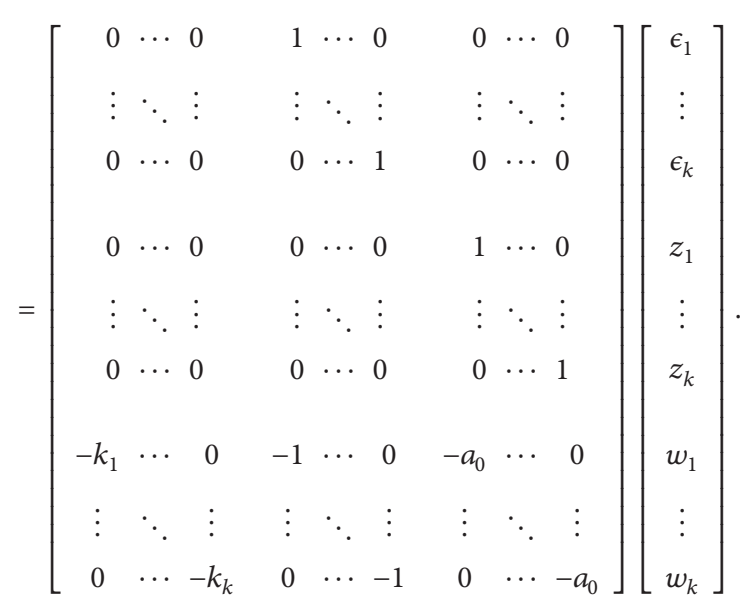

Theorem 5. Consider an array of Sprott circuits defined by (3) and (4), whose interconnections are established by the functionals (11) with the coupling signals given in (17). There exists a matrix $K$ that guarantees the asymptotic synchronization of the array defined by (8).

Proof. Equation (20) defines a nominal system formed by a set of uncoupled linear systems given by

$$
\left[\begin{array}{c}
\dot{\epsilon}_{i} \\
\dot{z}_{i} \\
\dot{w}_{i}
\end{array}\right]=\left[\begin{array}{ccc}
0 & 1 & 0 \\
0 & 0 & 1 \\
-k_{i} & -1 & -a_{0}
\end{array}\right]\left[\begin{array}{c}
\epsilon_{i} \\
z_{i} \\
w_{i}
\end{array}\right],
$$

for $i=1, \ldots, k$, whose characteristic polynomial is

$$
P(\lambda)=\lambda^{3}+a_{0} \lambda^{2}+\lambda+k_{i},
$$

and applying the Ruth-Hurwitz criterion, we obtain that the constant $k_{i}$ must satisfy

$$
0<k_{i}<a_{0}
$$

to guarantee exponentially asymptotic stability of the origin of system (21).

Now perturbed system (18) is analyzed. Considering that a state observer is designed such that the term $\mathscr{B}\left(\Gamma\left(x_{1}\right)-\right.$ $\left.\widetilde{\Gamma}\left(x_{1}\right)\right)+\mathscr{A}\left(\widetilde{\gamma}_{r}\left(x_{1_{r}}\right)-\gamma_{r}\left(x_{1_{r}}\right)\right)$ satisfies the vanish conditions established in [18], then the closed loop system (18) will have a globally asymptotically stable equilibrium point at the origin, which is why the synchronization problem defined by (8) is resolved.

In the following subsection, a proposal for an observer and a filter for perturbation estimation is presented.

3.1. State Observer for Disturbances Estimation. Consider the scalar system defined by the following equation:

$$
\begin{aligned}
& \dot{x}=f(x)+\gamma(x, t)+u, \\
& y=x,
\end{aligned}
$$

where $x \in \Re$ is the state of the system; $f(x)$ is a well-known function which satisfies the Lipschitz condition; $\gamma(x, t)$ is an unknown but bounded perturbation term; $|\gamma(x, t)|<\delta$. $u$ is a known entrance and the output is given by $y$. An observer for system (24) is as follows:

$$
\begin{aligned}
& \dot{\hat{x}}=f(x)+u+c_{1}(y-\widehat{y})+c_{2} \operatorname{sign}(y-\widehat{y}), \\
& \hat{y}=\widehat{x} .
\end{aligned}
$$

To demonstrate the convergence of the state of the observer (25) to the state of the plant (24), an error variable is defined $e=y-\widehat{y}$, whose dynamics are given by

$$
\dot{e}=\gamma(x, t)-c_{1} e-c_{2} \operatorname{sign}(e) .
$$

The right side of (26) is discontinuous, which is why the conditions for the existence of sliding mode on $s=e=0$ are analyzed.

The convergence of the trajectories of system (26) to $s=0$ is verified through the derivative of the function

$$
\begin{aligned}
V & =\frac{1}{2} s^{2}, \\
\dot{V} & =e \gamma(x, t)-c_{1} e^{2}-c_{2}|e|, \\
& \leq-c_{1} e^{2}-\left(c_{2}-|\gamma(x, t)|\right)|e| ;
\end{aligned}
$$

if

$$
c_{2}>\delta
$$

then

$$
\dot{V}<0
$$

which is why the trajectories converge to $e=0$ for any initial condition. Now we use the criterion given in [19] to ensure finite time convergence to the discontinuity surface.

$$
\begin{aligned}
& \lim _{s \rightarrow 0^{+}} \dot{s}<0, \\
& \lim _{s \rightarrow 0-} \dot{s}>0
\end{aligned}
$$

in this case, because $c_{2}>\delta$, we have

$$
\begin{aligned}
& \lim _{s \rightarrow 0^{+}} \dot{s}=\gamma(x, t)-c_{2}<0, \\
& \lim _{s \rightarrow 0^{-}} \dot{s}=\gamma(x, t)+c_{2}>0,
\end{aligned}
$$

which implies that the state of the observer converges the state of the plant in finite time.

Now, based on [19], we found the control that appears when the trajectories remain at $s=0$, which is called equivalent control $u_{\text {eq }}$.

Consider

$$
\dot{s}=\gamma(x, t)-u_{\mathrm{eq}}=0 ;
$$

therefore

$$
u_{\mathrm{eq}}=\gamma(x, t) \text {, }
$$


and the equivalent control $u_{\mathrm{eq}}$ is equal to the disturbances and it can be recovered through a low pass filter as indicated in [20].

$$
\dot{x}_{f}=-\alpha x_{f}+\alpha\left(c_{2} \operatorname{sign}(e)\right) \text {. }
$$

By using the observer (25) and the filter (34) to estimate the perturbations in the nodes and reference system, the conditions established in [18] are satisfied since equivalent control $u_{\text {eq }}$ is presented in finite time and, in theory, it can be recuperated in exact form using a low pass filter [19].

\section{Synchronization Technique Performance}

In this section, the performance of the synchronization technique is shown through a real-time simulation where an array formed by four nodes (3) and a reference system (4) is synchronized. The parameters of each system are in Table 1.

The array has the full chain interconnection scheme shown in Figure 1; therefore $\beta_{i, j}=\alpha_{i}=1$, for $i, j=1,2,3,4$. This way the functionals are as follows:

$$
\begin{aligned}
& \epsilon_{1}=e_{1, r}+e_{1,2}+e_{1,3}+e_{1,4}, \\
& \epsilon_{2}=e_{2, r}+e_{2,1}+e_{2,3}+e_{2,4}, \\
& \epsilon_{3}=e_{3, r}+e_{3,1}+e_{3,2}+e_{3,4}, \\
& \epsilon_{4}=e_{4, r}+e_{4,1}+e_{4,2}+e_{4,3},
\end{aligned}
$$

where the matrices $\mathscr{A}, \mathscr{B}, \mathscr{K}, G_{l}\left(x_{1}\right), G_{l_{r}}\left(x_{1_{r}}\right)$ are

$$
\begin{aligned}
\mathscr{A} & =\left[\begin{array}{llll}
1 & 1 & 1 & 1
\end{array}\right]^{T}, \\
\mathscr{B} & =\left[\begin{array}{rrrr}
4 & -1 & -1 & -1 \\
-1 & 4 & -1 & -1 \\
-1 & -1 & 4 & -1 \\
-1 & -1 & -1 & 4
\end{array}\right], \\
K & =\operatorname{diag}\{0.3\}, \\
G_{l}\left(x_{1}\right) & =\left[\begin{array}{llll}
G_{a_{1}} & G_{b_{2}} & G_{\mathcal{C}_{3}} & G_{d_{4}}
\end{array}\right]^{T} \\
G_{l_{r}}\left(x_{1_{r}}\right) & =G_{d_{r}} .
\end{aligned}
$$

The disturbance terms produced by parametric variations are estimated by an observer and a filter in each node and reference system. For the node $\Sigma_{1}$, the observer and the filter are

$$
\begin{aligned}
\dot{\hat{x}}_{3_{1}}= & -0.6 x_{3_{1}}-x_{2_{1}}+\left|x_{1_{1}}\right|-2+u_{1}+\left(x_{3_{1}}-\widehat{x}_{3_{1}}\right) \\
& +2 \operatorname{sign}\left(x_{3_{1}}-\widehat{x}_{3_{1}}\right), \\
\dot{x}_{f_{1}}= & -5 x_{f_{1}}+5\left(2 \operatorname{sign}\left(x_{3_{1}}-\widehat{x}_{3_{1}}\right)\right) ;
\end{aligned}
$$

for the node $\Sigma_{2}$

$$
\begin{aligned}
\dot{\hat{x}}_{3_{2}}= & -0.6 x_{3_{2}}-x_{2_{2}}-6 \max \left(x_{1_{2}}, 0\right)+0.5+u_{2} \\
& +\left(x_{3_{2}}-\widehat{x}_{3_{2}}\right)+2 \operatorname{sign}\left(x_{3_{2}}-\widehat{x}_{3_{2}}\right), \\
\dot{x}_{f_{2}}= & -5 x_{f_{2}}+5\left(2 \operatorname{sign}\left(x_{3_{2}}-\widehat{x}_{3_{2}}\right)\right) ;
\end{aligned}
$$

the observer and filter for the node $\Sigma_{3}$ are

$$
\begin{aligned}
\dot{\hat{x}}_{3_{3}}= & -0.6 x_{3_{2}}-x_{2_{2}}+1.2 x_{1_{3}}-4.5 \operatorname{sign}\left(x_{1_{3}}\right)+u_{3} \\
& +\left(x_{3_{3}}-\widehat{x}_{3_{3}}\right)+2 \operatorname{sign}\left(x_{3_{3}}-\widehat{x}_{3_{3}}\right), \\
\dot{x}_{f_{3}}= & -5 x_{f_{3}}+5\left(2 \operatorname{sign}\left(x_{3_{3}}-\widehat{x}_{3_{3}}\right)\right) ;
\end{aligned}
$$

for the node $\Sigma_{4}$

$$
\begin{aligned}
\dot{\hat{x}}_{3_{4}}= & -0.6 x_{3_{4}}-x_{2_{4}}-1.2 x_{1_{4}}+2 \operatorname{sign}\left(x_{1_{4}}\right)+u_{4} \\
& +\left(x_{3_{4}}-\widehat{x}_{3_{4}}\right)+2 \operatorname{sign}\left(x_{3_{4}}-\widehat{x}_{3_{4}}\right), \\
\dot{x}_{f_{4}}= & -5 x_{f_{4}}+5\left(2 \operatorname{sign}\left(x_{3_{4}}-\widehat{x}_{3_{4}}\right)\right) ;
\end{aligned}
$$

finally, for the reference system $\Sigma_{r}$

$$
\begin{aligned}
\dot{\hat{x}}_{3_{r}}= & -0.6 x_{3_{r}}-x_{2_{r}}-1.2 x_{1_{r}}+2 \operatorname{sign}\left(x_{1_{r}}\right) \\
& +\left(x_{3_{r}}-\widehat{x}_{3_{r}}\right)+2 \operatorname{sign}\left(x_{3_{r}}-\widehat{x}_{3_{r}}\right), \\
\dot{x}_{f_{r}}= & -5 x_{f_{r}}+5\left(2 \operatorname{sign}\left(x_{3_{r}}-\widehat{x}_{3_{r}}\right)\right) .
\end{aligned}
$$

This way the vectors of estimated perturbations are defined as follows:

$$
\begin{aligned}
\widetilde{\Gamma}(\cdot) & =\left[\begin{array}{llll}
x_{f_{1}} & x_{f_{2}} & x_{f_{3}} & x_{f_{4}}
\end{array}\right]^{T}, \\
\gamma_{r}(\cdot) & =x_{f_{r}} .
\end{aligned}
$$

With these elements, the vector of coupling signals (17) is implemented.

The array and the coupling signals were implemented in a $\mathrm{dSPACE}^{\circledR} 1103$ real-time control platform through Simulink ${ }^{\circledR}$ from Matlab ${ }^{\circledR}$. The algorithm odel (Euler) fixed step, with a sample time of 0.0001 seconds, was used to carry out the experiment. The results are described as follows.

In Figure 2, the actual and identified perturbations, in each node and reference system, are shown. Since it is a numerical experiment, it is possible to compare the actual perturbations produced by parametric variations, shown in Table 1, with the one estimated perturbation. As can be observed in the said figure, both graphs overlap, which is why perturbation identification is efficient. Graphs (a), (b), (c), and (d) correspond to the perturbations in systems $\Sigma_{1}, \Sigma_{2}, \Sigma_{3}$, and $\Sigma_{4}$, respectively, while graph (e) corresponds to the reference system $\Sigma_{r}$, which is zero because there are not parametric variations.

In Figure 3, coupling signals for each node are shown. Here the time starts at $t=100 \mathrm{sec}$ because at this time the coupling signals are applied. As can be observed, such signals 
TABLE 1: Parameters of the nodes and the reference system.

\begin{tabular}{lllccccc}
\hline System & $G_{l}$ & $a_{0}$ & $\Delta_{a}$ & $b_{0}$ & $\Delta_{b}$ & $c_{0}$ \\
\hline$\Sigma_{1}$ & $G_{a}$ & 0.6 & 0.006 & 1 & 0 & 0 & 2 \\
$\Sigma_{2}$ & $G_{b}$ & 0.6 & 0.006 & 6 & 1.2 & 0.012 & 0.5 \\
$\Sigma_{3}$ & $G_{c}$ & 0.6 & 0 & -1.2 & -0.012 & 4.5 & 0 \\
$\Sigma_{4}$ & $G_{d}$ & 0.6 & 0.006 & -1.2 & 0 & 2 \\
$\Sigma_{r}$ & $G_{d}$ & 0.6 & 0 & & 0 & 0 \\
\hline
\end{tabular}

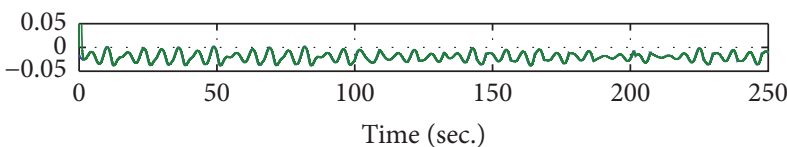

(a)

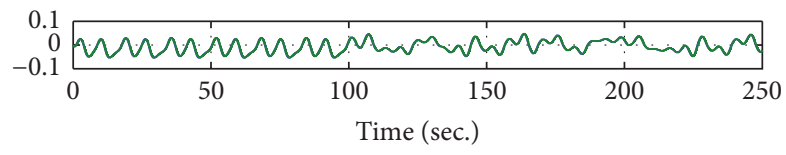

(c)

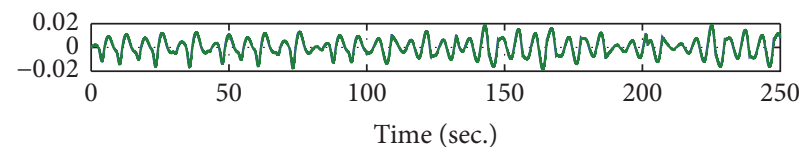

(b)

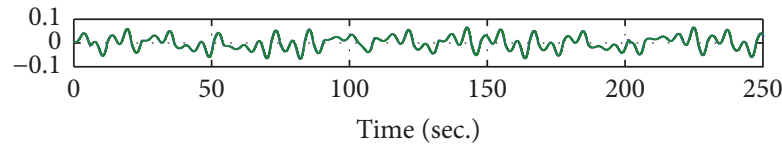

(d)

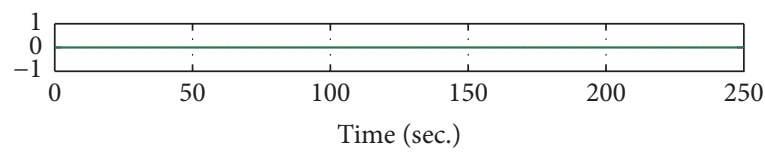

(e)

FIGURE 2: Actual and estimated disturbances in each node and reference system.

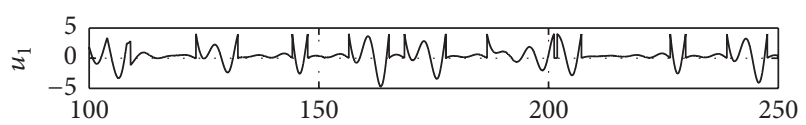

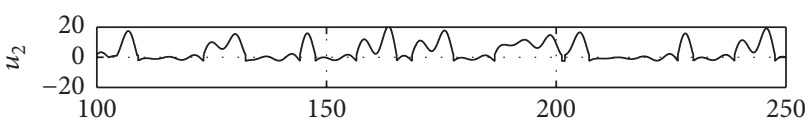

$=\underbrace{10}_{100} \underbrace{}_{150}$

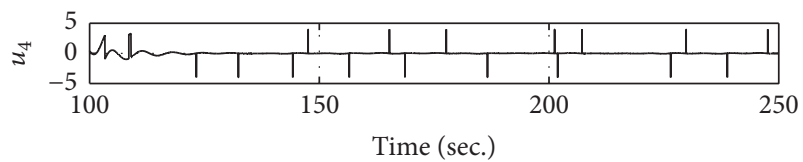

Figure 3: Coupling signals in each node.

do not contain high frequency components as when a sliding mode technique is used.

Figure 4 shows the output for each node and reference systems. When the coupling signals are not applied, in the first 100 seconds, the systems evolve freely. In $t=100 \mathrm{sec}$, as the dashed line indicates, the coupling signals are applied and the output of each node converges asymptotically to the output of the reference system. Finally, in Figure 5, the attractors of each node before applying the coupling signals (left) and after applying the said signals (right) are shown; the attractor of each node transforms into the reference system attractor.

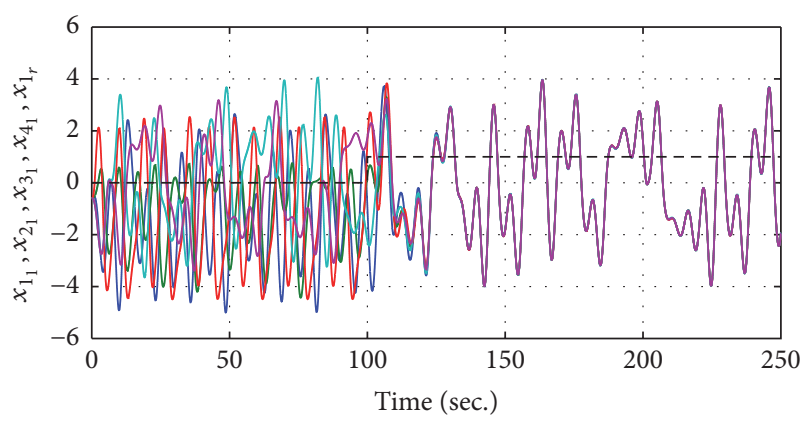

FIGURE 4: Outputs of each node and reference system before and after applying coupling signals.

\section{Conclusions}

The synchronization technique proposed in this paper can be applied to different kinds of arrays of Sprott circuits like master-slave, rings, and trees. Furthermore, by means of the individual connection sense, the unidirectional or bidirectional couplings among the nodes conforming the array can be established. This technique does not impose a symmetric structure of the connectivity matrix and uses a robust observer and a filter that estimates the disturbances due to parametric uncertainties; therefore the closed loop is robust.

The application of the proposed synchronization method to an array with four nodes and a reference system on a real-time platform showed a good robustness, even when 


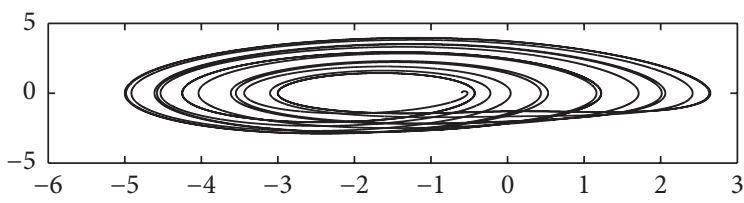

(a)

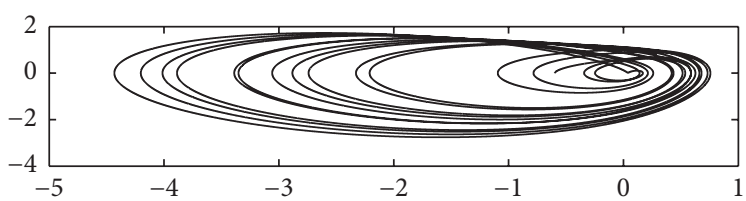

(c)

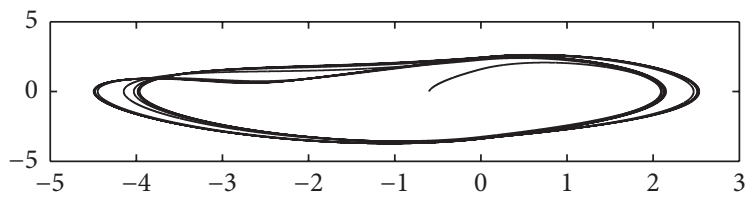

(e)

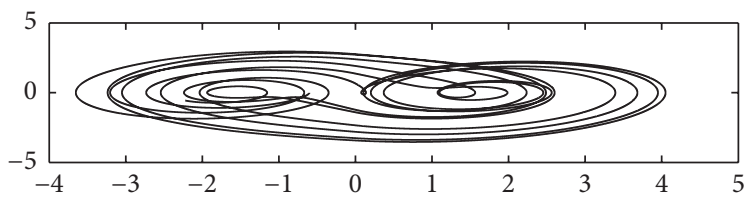

(g)

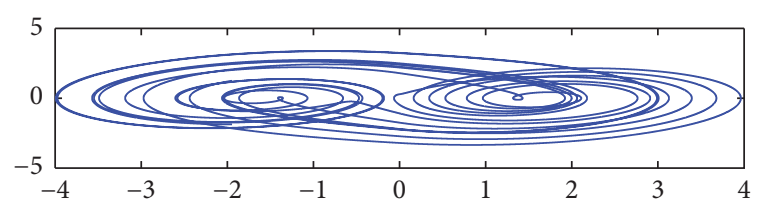

(b)

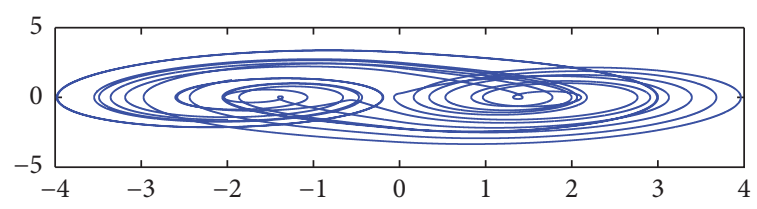

(d)

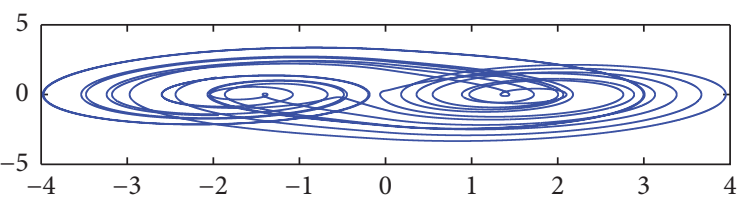

(f)

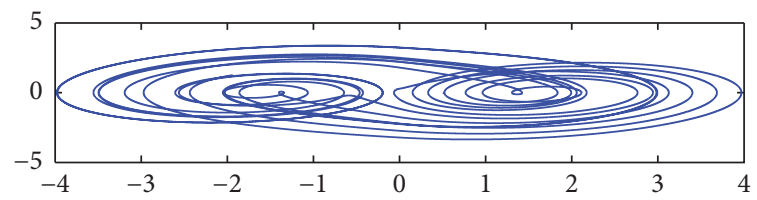

(h)

FIGURE 5: Projection in the plain $\left(x_{1_{i}}, x_{2_{i}}\right)$ of the phase portrait of each node of the array before (left hand) and after (right hand) applying coupling signals.

there were parametric uncertainties that produce nonsmooth terms.

The main limitation of the proposed synchronization technique is that it needs a real-time control platform, such as the dSPACE 1103 system, for its implementation, since the accuracy of the identification of the disturbances depends on the sampling time and its periodicity.

In this way, the future work is the implementation of Sprott circuit array using analog circuits, both nodes and coupling signals, in order to avoid the use of a real-time control platform.

\section{Conflicts of Interest}

The authors declare that they have no conflicts of interest.

\section{References}

[1] A. Pikovsky, M. Rosenblum, and J. Kurths, Synchronization, a uNiversal Concept in Nonlinear Sciences, vol. 12, Cambridge University Press, 2003.

[2] I. I. Blekhman, A. L. Fradkov, H. Nijmeijer, and A. Y. Pogromsky, "On self-synchronization and controlled synchronization," Systems and Control Letters, vol. 31, no. 5, pp. 299-305, 1997.

[3] L. M. Pecora and T. L. Carroll, "Synchronization in chaotic systems," Physical Review Letters, vol. 64, no. 8, pp. 821-824, 1990.
[4] Y.-Y. Hou, H.-C. Chen, J.-F. Chang, J.-J. Yan, and T.-L. Liao, "Design and implementation of the Sprott chaotic secure digital communication systems," Applied Mathematics and Computation, vol. 218, no. 24, pp. 11799-11805, 2012.

[5] J.-S. Lin, C.-F. Huang, T.-L. Liao, and J.-J. Yan, "Design and implementation of digital secure communication based on synchronized chaotic systems," Digital Signal Processing, vol. 20, no. 1, pp. 229-237, 2010.

[6] D. Chen, R. Zhang, J. C. Sprott, H. Chen, and X. Ma, "Synchronization between integer-order chaotic systems and a class of fractional-order chaotic systems via sliding mode control," Chaos: An Interdisciplinary Journal of Nonlinear Science, vol. 22, no. 2, article 023130, 2012.

[7] S. Liu and F. Zhang, "Complex function projective synchronization of complex chaotic system and its applications in secure communication," Nonlinear Dynamics, vol. 76, no. 2, pp. 10871097, 2014.

[8] B. Li, "Finite-time synchronization for complex dynamical networks with hybrid coupling and time-varying delay," Nonlinear Dynamics, vol. 76, no. 2, pp. 1603-1610, 2014.

[9] B. R. Andrievsky and A. L. Fradkov, "Adaptive synchronization in presence of noise with application to telecommunications," in Proceedings of the 6th European Control Conference, ECC 2001, vol. 2953, pp. 2953-2957, September 2001.

[10] J. C. Sprott, "Simple chaotic systems and circuits," American Journal of Physics, vol. 68, no. 8, pp. 758-763, 2000.

[11] X. Wang and Y. Wang, "Adaptive control for synchronization of a four-dimensional chaotic system via a single variable," Nonlinear Dynamics, vol. 65, no. 3, pp. 311-316, 2011. 
[12] I. Belykh, M. Hasler, M. Lauret, and H. Nijmeijer, "Synchronization and graph topology," International Journal of Bifurcation and Chaos, vol. 15, no. 11, pp. 3423-3433, 2005.

[13] C. Sarasola, F. J. Torrealdea, A. D’Anjou, A. Moujahid, and M. Graña, "Feedback synchronization of chaotic systems," International Journal of Bifurcation and Chaos, vol. 13, no. 1, pp. 177-191, 2003.

[14] S. J. Wang, N. S. Pai, and H. T. Yau, "Robust controller design for synchronization of two chaotic circuits," Information Technology Journal, vol. 8, pp. 743-749, 2009.

[15] X. Xiao-dong, Y. Wan-li, and Z. Su-wen, "Finite time synchronization of Sprott circuits with uncertain parameters," in Proceedings of the International Conference on Advanced Computer Control, ICACC'09, pp. 693-696, 2009.

[16] D. Rosas, J. Alvarez, and E. Alvarez, "Robust synchronization of arrays of uncertain nonlinear second-order dynamical systems," Nonlinear Dynamics, vol. 67, no. 4, pp. 2735-2746, 2012.

[17] J. Alvarez, D. Rosas, D. Hernandez, and E. Alvarez, "Robust synchronization of arrays of Lagrangian systems," International Journal of Control, Automation and Systems, vol. 8, no. 5, pp. 1039-1047, 2010.

[18] H. K. Khalil and J. W. Grizzle, Nonlinear Systems, vol. 3, Prentice Hall, New Jersey, NJ, USA, 1996.

[19] V. I. Utkin, Sliding Modes and Their Application in Variable Structure Systems, Mir Publishers, 1978.

[20] V. Utkin, J. Guldner, and J. Shi, Sliding mode control in electromechanical systems, vol. 34, CRC Press, Boca Raton, FL, USA, 2009. 


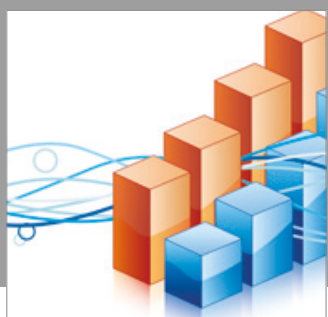

Advances in

Operations Research

vatersals

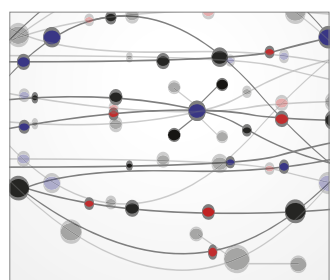

\section{The Scientific} World Journal
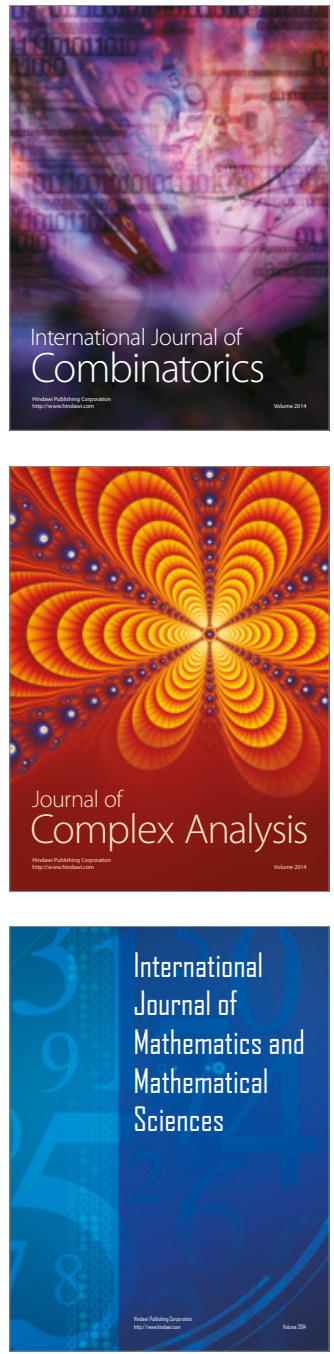
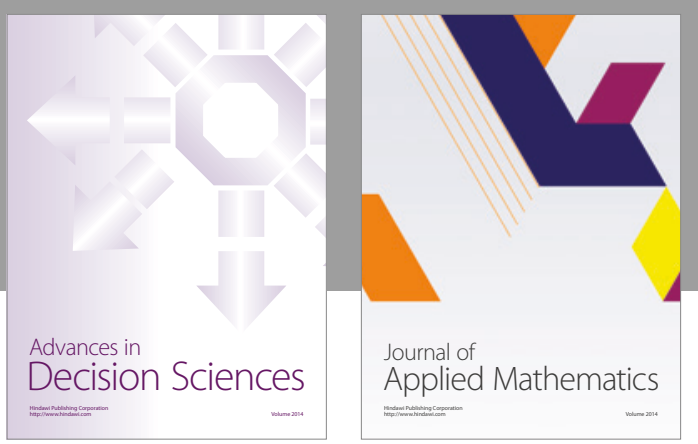

Algebra

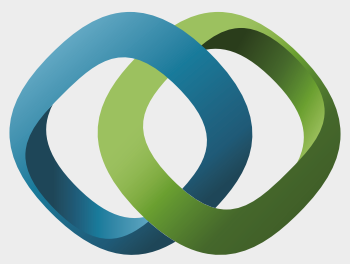

\section{Hindawi}

Submit your manuscripts at

https://www.hindawi.com
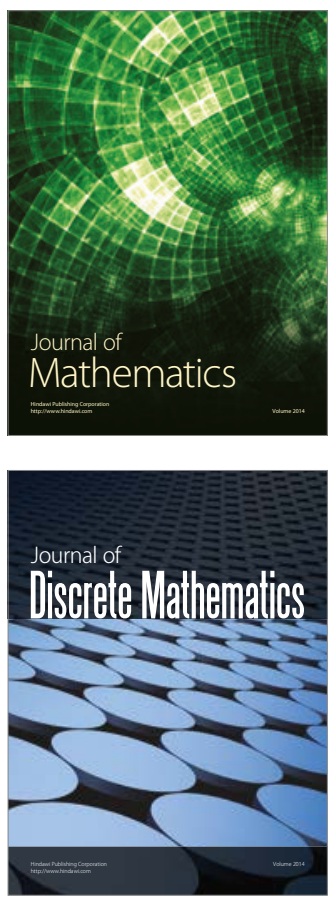

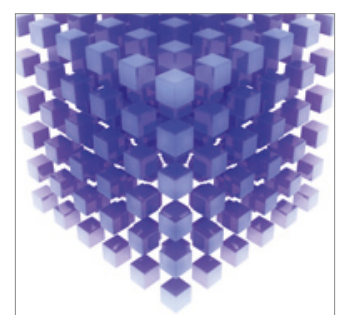

Mathematical Problems in Engineering
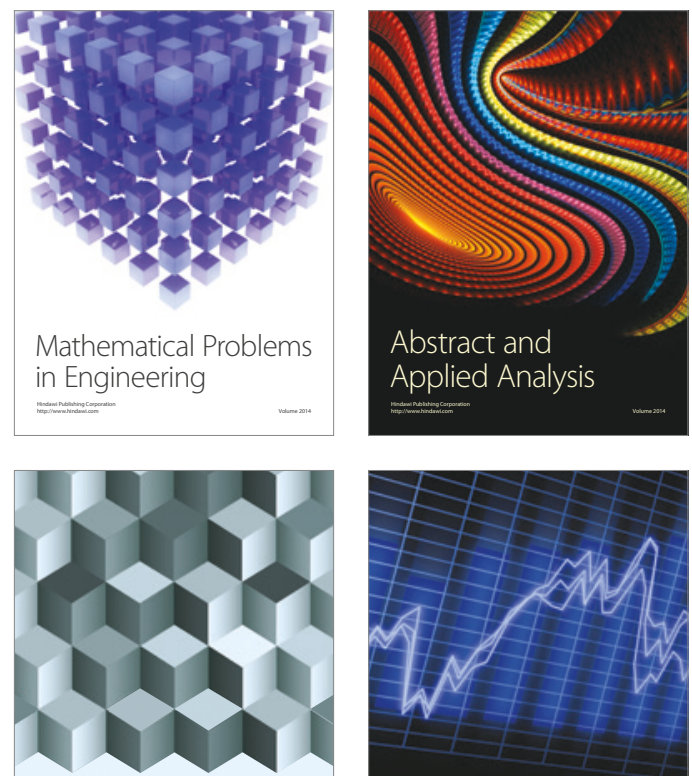

Journal of

Function Spaces

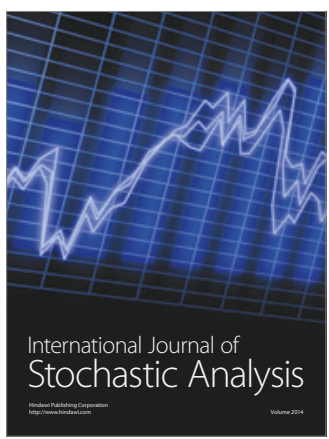

Probability and Statistics
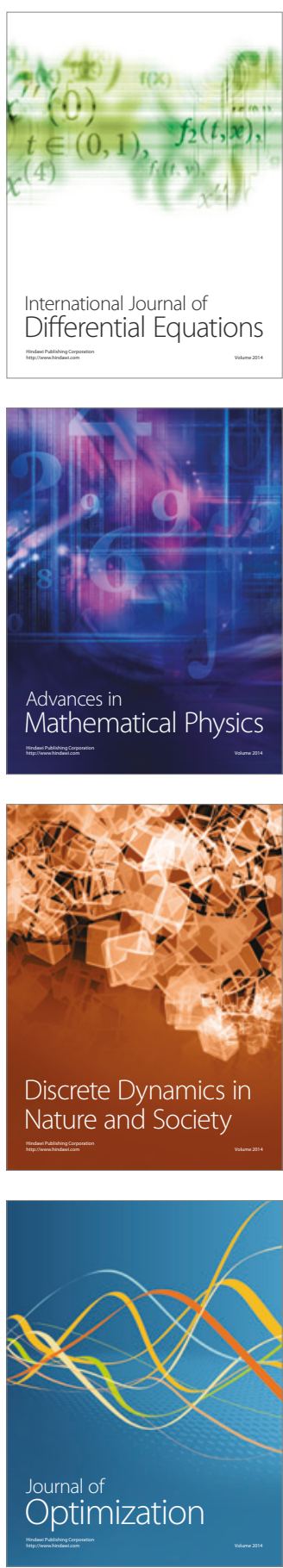